\title{
Directed flow of charged particles within idealized viscous hydrodynamics at energies available at the BNL Relativistic Heavy Ion Collider and at the CERN Large Hadron Collider
}

\author{
Ze-Fang Jiang $\odot,{ }^{1,2,3, *}$ C. B. Yang $\odot,,^{2,3, \dagger}$ and Qi Peng ${ }^{1}$ \\ ${ }^{1}$ Department of Physics and Electronic-Information Engineering, Hubei Engineering University, Xiaogan 432000, China \\ ${ }^{2}$ Institute of Particle Physics, Central China Normal University, Wuhan 430079, China \\ ${ }^{3}$ Key Laboratory of Quark and Lepton Physics, Ministry of Education (MOE), Wuhan 430079, China
}

(Received 22 January 2021; revised 7 July 2021; accepted 25 October 2021; published 8 December 2021)

\begin{abstract}
Following the Bożek-Wyskiel parametrization tilted initial condition, an alternative way to construct a longitudinal tilted fireball based on the Glauber collision geometry is presented. This longitudinal tilted initial condition combined with the Ideal-CLVisc $(3+1) \mathrm{D}$ hydrodynamic model, a nonvanishing directed flow coefficient $v_{1}$ in a wide range is observed. After comparing the model's results with experimentally observed data of directed flow coefficient $v_{1}(\eta)$ from $\sqrt{s_{N N}}=200 \mathrm{GeV} \mathrm{Cu}+\mathrm{Cu}, \mathrm{Au}+\mathrm{Au}$ collisions at RHIC energy to $\sqrt{s_{N N}}=2.76 \mathrm{TeV}$ and $\sqrt{s_{N N}}=5.02 \mathrm{TeV} \mathrm{Pb}+\mathrm{Pb}$ collisions at the LHC energy. One finds that directed flow measurements in heavy-ion collisions can set strong constraints on the imbalance of forward and backward incoming nuclei and on the magnitude asymmetry of pressure gradients along the $x$ direction.
\end{abstract}

DOI: 10.1103/PhysRevC.104.064903

\section{INTRODUCTION}

In the past decades, relativistic heavy-ion collisions provided methods to explore and understand the deconfined quark-gluon plasma (QGP). The strongly collective flow of such a hot dense medium is one of the key observations in the physics of high-energy heavy-ion collisions [1-4].

The rapidity-odd directed flow (shorted as directed flow or $v_{1}$ ) refers to the collective sideward deflection of particles and is the first-order harmonic of the Fourier expansion of the particle azimuthal distribution with respect to the reaction plane $[5,6]$. The directed flow is believed to be created at the very early stage (during the nuclear passage time: $2 R / \gamma \approx 0.1$ $\mathrm{fm} / c$ ) at large rapidity $\eta$ (in the fragmentation region) [7]. Therefore, it may keep trace of the bulk collective dynamics and the subsequent evolution into a thermalized hot QCD matter, which provides a unique insight to investigate the initial condition.

At the current stage, the directed flow $v_{1}$ and the splitting $\Delta v_{1}$ between particles and antiparticles are measured for both light charged hadrons and heavy quark productions (e.g., $J / \psi$, $D^{0}, \bar{D}^{0}$ ) at the BNL Relativistic Heavy Ion Collider (RHIC) and at the Large Hadron Collider (LHC) [8-12]. A striking characteristic of the measured directed flow for charged particle is the large value of $v_{1}$ at RHIC energy [8] and large value of splitting $\Delta v_{1}$ of charm hadrons produced at LHC energy

\footnotetext{
*jiangzf@mails.ccnu.edu.cn

†cbyang@mail.ccnu.edu.cn
}

Published by the American Physical Society under the terms of the Creative Commons Attribution 4.0 International license. Further distribution of this work must maintain attribution to the author(s) and the published article's title, journal citation, and DOI. Funded by $S C O A P^{3}$.
[11]. The directed flow has been investigated by different models and mechanisms, such as the transport model [13], three-dimensional (3D) initial geometric asymmetry source + hydrodynamic model [4,14-21], extremely strong magneticfield effect [20,22-26], the chiral magnetic effect (CME) and magnetohydrodynamics (MHD) [27], vorticity effect [28], AMPT + quark coalescence model [29], and so on.

The CCNU-LBNL-viscous hydrodynamic (CLVisc) model [31] is an open source $(3+1)$-dimensional [ $(3+1) \mathrm{D}]$ hydrodynamic frame for heavy ion collisions that was developed by Pang et al. [30], which is parallelized on a graphics processing unit (GPU) using the Open Computing Language (OpenCL). There is tremendous progress in understanding the QCD matter by using the CLVisc model, such as the strong vorticity prediction [32], which was later found at the RHIC-STAR [33], the magnetic-field-induced squeezing effect calculation [34], the description for longitudinal decorrelation of anisotropic flow $[35,36]$, deep learning, and machine learning coupled with heavy ion collisions [37], and the jet quenching research $[38,39]$. The original configurations and code of CLVisc $(3+1) \mathrm{D}$ hydrodynamics can be downloaded available in gitlab website [41].

In this paper, following the well-known Bożek-Wyskiel parametrization tilted initial condition in Refs. [15,21,27,40], an alternative parametrization to generate the tilted longitudinal structure of the fireball is presented. The contribution of the forward-going and backward-going participant nucleons is assumed to be imbalance and related to a phenomenological parameter $H_{t}$ in the Glauber model. Such a modified initial condition with the Ideal-CLVisc $(3+1)$ D hydrodynamics simulation give a finite directed flow of the charged particles (and $\pi^{+}$) in the middle and backward and forward rapidity region compared with the STAR and ALICE measurements.

The article is organized as follows: In Sec. II, the parametrization and the modified initial condition for the 
TABLE I. Table of parameters used in the Woods-Saxon distribution for $\mathrm{Cu}, \mathrm{Au}$, and $\mathrm{Pb}$ described in the text [43].

\begin{tabular}{lcccc}
\hline \hline Nucleus & $A$ & $n_{0}\left[1 / \mathrm{fm}^{3}\right]$ & $R[\mathrm{fm}]$ & $d[\mathrm{fm}]$ \\
\hline $\mathrm{Cu}$ & 63 & 0.17 & 4.20 & 0.546 \\
$\mathrm{Au}$ & 197 & 0.17 & 6.38 & 0.546 \\
$\mathrm{~Pb}$ & 208 & 0.17 & 6.62 & 0.535 \\
\hline \hline
\end{tabular}

CLVisc (3+1)D ideal hydrodynamic simulation are presented. In Sec. III, our numerical results on the directed flow of charged particles are presented. Finally, in Sec. IV, we summarize the results and present a short outlook.

\section{SETUP OF THE NUMERICAL SIMULATIONS}

In this work, following Refs. [15,21,27], the viscous corrections are not included in the present study ${ }^{1}$ and the viscosity effect will be included in future studies. The $(3+$ 1)-dimensional numerical simulations are performed with in Milne/Bjorken coordinates.

\section{A. Initial condition}

The initial energy density distribution is computed according to a modified optical Glauber model [31,42].

In the optical Glauber model, the nucleus thickness function $T(x, y)$ from the Woods-Saxon distribution is

$$
T(x, y)=\int_{-\infty}^{\infty} d z \frac{n_{0}}{1+e^{\left(\sqrt{x^{2}+y^{2}+z^{2}}-R\right) / d}},
$$

where $n_{0}$ is the average nuclear density, $d$ is the diffusiveness, $x, y, z$ is the space coordinates and $R$ is the radius of the nuclear Fermi distribution, which depends on the specific nucleus. The parameters used for nucleus $\mathrm{Cu}, \mathrm{Au}$, and $\mathrm{Pb}$ in current study are listed in Table I.

$T_{1}\left(\mathbf{x}_{T}\right)$ and $T_{2}\left(\mathbf{x}_{T}\right)$ are the densities of participants from the two nuclei,

$$
\begin{aligned}
& T_{1}\left(\mathbf{x}_{T}\right)=T_{+}\left(\mathbf{x}_{T}\right)\left[1-\left(1-\frac{\sigma_{N N} T_{-}\left(\mathbf{x}_{T}\right)}{A}\right)^{A}\right], \\
& T_{2}\left(\mathbf{x}_{T}\right)=T_{-}\left(\mathbf{x}_{T}\right)\left[1-\left(1-\frac{\sigma_{N N} T_{+}\left(\mathbf{x}_{T}\right)}{A}\right)^{A}\right],
\end{aligned}
$$

where $A$ is the mass number of the colliding nuclei, $\sigma_{N N}$ is the inelastic-scattering cross section, $\sigma_{N N}$ are set to $40 \mathrm{mb}$ for $\sqrt{s_{N N}}=200 \mathrm{GeV} \mathrm{Cu}+\mathrm{Cu}, \mathrm{Au}+\mathrm{Au}, 64 \mathrm{mb}$ for $\sqrt{s_{N N}}=$ 2.76 TeV Pb $+\mathrm{Pb}, 67 \mathrm{mb}$ for $\mathrm{Pb}+\mathrm{Pb} \sqrt{s_{N N}}=5.02 \mathrm{TeV}$ collisions [43], and

$$
T_{+}\left(\mathbf{x}_{T}\right)=T\left(\mathbf{x}_{T}+\mathbf{b} / 2\right), \quad T_{-}\left(\mathbf{x}_{T}\right)=T\left(\mathbf{x}_{T}-\mathbf{b} / 2\right),
$$

where $\mathbf{x}_{T}=(x, y)$ is the vector of the transverse plane coordinates and $\mathbf{b}$ is the impact-parameter vector, connecting the centers of the two nuclei.

\footnotetext{
${ }^{1}$ The directed flow is generated at very early stage in the evolution and it is given by the initial tilted source. The ideal fluid approximation could give a sizable directed flow coefficient $v_{1}$ and directly reflect the evolution of pressure [15].
}

TABLE II. Maximum energy density for CLVisc $(3+1) \mathrm{D}$ ideal hydrodynamics starting from initial proper time $\tau_{0}=0.2 \mathrm{fm}$ to reproduce the charged multiplicity distribution at RHIC and at the LHC [34].

\begin{tabular}{lcc}
\hline \hline System & $\sqrt{s_{N N}}$ & $\varepsilon_{0}\left[\mathrm{GeV} / \mathrm{fm}^{3}\right]$ \\
\hline $\mathrm{Cu}+\mathrm{Cu}$ & $200 \mathrm{GeV}$ & 83.5 \\
$\mathrm{Au}+\mathrm{Au}$ & $200 \mathrm{GeV}$ & 155.5 \\
$\mathrm{~Pb}+\mathrm{Pb}$ & $2.76 \mathrm{TeV}$ & 465.0 \\
$\mathrm{~Pb}+\mathrm{Pb}$ & $5.02 \mathrm{TeV}$ & 580.0 \\
\hline \hline
\end{tabular}

The function $W_{N}$ gives the contribution of the wounded nucleons. To generate a tilted fireball along the longitudinal direction, the wounded nucleons [42] weight function $W_{N}$ is modified as follows:

$$
\begin{aligned}
W_{N}\left(x, y, \eta_{s}\right)= & {\left[T_{1}(x, y)+T_{2}(x, y)\right]+H_{t}\left[T_{2}(x, y)\right.} \\
& \left.-T_{1}(x, y)\right] \tan \left(\frac{\eta_{s}}{\eta_{t}}\right),
\end{aligned}
$$

here the longitudinal tilted parameter $H_{t}$ is a free parameter that reflects the imbalance in the emitting contributions from forward-going and backward-going participant nucleons. As we will see in the following section, varying the parameter $H_{t}$ results in strong dependencies in the magnitude of the pion's and charged particle's directed flow. The parameter $\eta_{t}$ is a constant and chosen to be 8.0 for all the collision systems.

The energy density distribution at the hydrodynamic starting time $\tau_{0}$ is given by

$$
\varepsilon\left(x, y, \eta_{s}\right)=\varepsilon_{0} W\left(x, y, \eta_{s}\right) H\left(\eta_{s}\right),
$$

where $\varepsilon_{0}$ the maximum energy density given in Table II. For most-central collisions at RHIC and the LHC energy, the total weight function $W\left(x, y, \eta_{s}\right)$ is defined as [27,34]

$$
W\left(x, y, \eta_{s}\right)=\frac{(1-\alpha) W_{N}\left(x, y, \eta_{s}\right)+\alpha n_{B C}(x, y)}{(1-\alpha) W_{N}(0,0,0)+\left.\alpha n_{B C}(0,0)\right|_{\mathbf{b}=0}},
$$

with $\alpha=0.05$ being the collision hardness parameter ${ }^{2}$ and $n_{B C}(x, y)$ being the mean number of binary collisions:

$$
n_{B C}(x, y)=\sigma_{N N} T_{+}(x, y) T_{-}(x, y) .
$$

The energy density profile in the longitudinal direction is modulated by [31]

$$
H\left(\eta_{s}\right)=\exp \left[-\frac{\left(\eta_{s}-\eta_{w}\right)^{2}}{2 \sigma_{\eta}^{2}} \theta\left(\eta_{s}-\eta_{w}\right)\right],
$$

while the parameters $\eta_{w}$ and $\sigma_{\eta}$ are set to 2.95 and 0.4 for $\mathrm{Au}+\mathrm{Au}$ and $\mathrm{Cu}+\mathrm{Cu} \sqrt{s_{N N}}=200 \mathrm{GeV}, 3.6$ and 0.6 for $\mathrm{Pb}+\mathrm{Pb} \sqrt{s_{N N}}=2.76 \mathrm{TeV}$, and 3.6 and 0.7 for $\mathrm{Pb}+\mathrm{Pb}$ $\sqrt{s_{N N}}=5.02 \mathrm{TeV}$ collisions. $\eta_{w}$ and $\sigma_{\eta}$ lead to a two-peak structure along the longitudinal direction in the final state $[27,31,34]$.

\footnotetext{
${ }^{2}$ The parameter $\alpha$ should be collision-energy dependent when reproducing the centrality dependence of multiplicity necessitates. In this paper we follow Refs. [27,34] and assume $\alpha=0.05$ for simplicity.
} 
TABLE III. Impact parameters $b$ used in the Glauber model for $\mathrm{Cu}+\mathrm{Cu}, \mathrm{Au}+\mathrm{Au}$, and $\mathrm{Pb}+\mathrm{Pb}$ described in the text [43].

\begin{tabular}{lccccc}
\hline \hline$b$ & $0 \%-5 \%$ & $10 \%-15 \%$ & $10 \%-20 \%$ & $30 \%-40 \%$ & $30 \%-60 \%$ \\
\hline $\mathrm{Cu}+\mathrm{Cu}$ & 1.74 & 3.70 & 4.03 & 6.17 & 6.85 \\
$\mathrm{Au}+\mathrm{Au}$ & 2.40 & 5.27 & 5.76 & 8.78 & 9.76 \\
$\mathrm{~Pb}+\mathrm{Pb}$ & 2.65 & 5.58 & 6.09 & 9.33 & 10.28 \\
\hline \hline
\end{tabular}

The collision centrality class is determined by the impact parameter $b$, which can be obtained by interpolation [43]. The impact parameter using in this paper is presented in Table III.

With the above parametrizations, we illustrate the profile of energy density and magnitude of pressured gradient distribution for $0 \%-5 \% \mathrm{Au}+\mathrm{Au}$ collisions at $\sqrt{s_{N N}}=200 \mathrm{GeV}$ and $10 \%-20 \% \mathrm{~Pb}+\mathrm{Pb}$ collisions at $\sqrt{s_{N N}}=2.76 \mathrm{TeV}$ in Figs. 1 and 2 .

Figure 1(a) shows the energy density distribution on the $\eta_{s}-x$ plane $(y=0.0 \mathrm{fm})$ at $\tau=0.2 \mathrm{fm}$ in centrality class $0 \%-5 \%(b=2.4 \mathrm{fm}) \mathrm{Au}+\mathrm{Au}$ collisions at $\sqrt{s_{N N}}=$ $200 \mathrm{GeV}$ with the tilted parameter $H_{t}=1$. It is evident that the parameter $H_{t}$ in Eq. (5) controls the imbalance in the forward-backward hemispheres and leads to a longitudinal tilted fireball.

Figures 1(b) and 1(c) show the magnitude of initial pressure gradients in the transverse plane at $\tau=0.2 \mathrm{fm}$ and forward-rapidity $\eta_{s}=2.1$. The magnitude of pressure gradient $\left(-\partial_{x} P\right.$ and $\left.-\partial_{y} P\right)$ is calculated from the initial energy density and equation of state (EoS). We find that the magnitude of the pressure gradient shows an asymmetry along the $x>0$ and $x<0$ direction in the transverse plane. The value of $-\partial_{x} P$ shows a maximal value of around $16 \mathrm{GeV} / \mathrm{fm}^{4}$ $\left(14 \mathrm{GeV} / \mathrm{fm}^{4}\right)$ at the $x>0(x<0)$ panel. With the hydrodynamic expansion, considering the contribution from the transverse and longitudinal pressures, the final directed flow coefficient becomes negative for positively rapidity $[15,40]$.

Figure 2(a) shows the initial energy density distribution on the $\eta_{s^{-}} x$ plane $(y=0.0 \mathrm{fm})$ at $\tau=0.2 \mathrm{fm}$ in $10 \%-20 \%(b=$ 6.09) $\mathrm{Pb}+\mathrm{Pb}$ collisions at $\sqrt{s_{N N}}=2.76 \mathrm{TeV}$ with the tilted parameter $H_{t}=0.70$.

Figures 2(b) and 2(c) show the magnitude of initial pressure gradients in the transverse plane at $\tau=0.2 \mathrm{fm}$ and forward rapidity $\eta_{s}=2.1$. The magnitude of pressure gradient $\left(-\partial_{x} P\right.$ and $\left.-\partial_{y} P\right)$ shows an asymmetry along the $x>0$ and $x<0$ direction. Furthermore, the value of $-\partial_{x} P$ shows a maximal value of around $48 \mathrm{GeV} / \mathrm{fm}^{4}\left(40 \mathrm{GeV} / \mathrm{fm}^{4}\right)$ along the $x>0(x<0)$ direction in the transverse plane.

The information on how the above initial spatial anisotropy is transferred to the momentum space $[15,40]$ is encoded on the directed flow coefficient, which will be presented in Sec. III.

\section{B. Hydrodynamic equations and simulations}

The hydrodynamic equations from the literature read [44-48]

$$
\partial_{\mu} T^{\mu \nu}=0,
$$

where $T^{\mu v}=\varepsilon u^{\mu} u^{v}-P \Delta^{\mu \nu}$ is the energy-momentum tensor for ideal hydrodynamics, $u^{\mu}=\gamma(1, \mathbf{v})$ denotes the fluid velocity four-vector, $\varepsilon$ is the energy density, and $P$ is the pressure.
The pressure $P$ is given as a function of energy density $\varepsilon$ by the equation of state $(\mathrm{EoS})$. The lattice QCD equation of state from the Wuppertal-Budapest group (2014) [49] is used in the current study. Notice that the viscosity and net baryon density are set to zero in the current study.

The energy-momentum conservation equations [Eq. (10)] are solved numerically by using Kurganov-Tadmor (KT) algorithm [31], which was introduced to the field of high-energy physics by the McGill group [50]. The 3D partial differential equations are solved by updating the values of fluid cells at each time step. For each collision system, we run the Ideal-CLVisc $(3+1) \mathrm{D}$ hydrodynamic simulation with number of cells $N_{\text {cells }}=N_{x} \times N_{y} \times N_{\eta_{s}}=201 \times 201 \times 105$ for 1600 time steps on GPU NVIDIA GeForce RTX 2080TI (Turing features) and server CPU Intel Xeon E5-1620v2. For more details about this GPU simulation part, see Refs. [31,51]. The freeze-out condition used in current calculation is the isothermal freeze-out condition [31], where one assumes the hypersurface is determined by a constant temperature $T_{f r z}=$ $137 \mathrm{MeV}$.

Based on the Cooper-Frye formula [52], the Ideal-CLVisc provided a "smooth method" to compute the different particle spectra on the freeze-out hypersurface, where the numerical integration is performed over the freeze-out hypersurface and smooth particle spectra are obtained in $N_{Y} \times N_{p t} \times N_{\phi}=$ $41 \times 15 \times 48$ tabulated $\left(Y, p_{T}, \phi\right)$ bins [31]. $p_{T}$ and $\phi$ are chosen to be Gaussian quadrature nodes to simplify the calculation of $p_{T}$ or $\phi$ integrated spectra. Hadron spectra from resonance decays are also computed via integration and parallelized on the GPU.

\section{Rapidity-odd directed flow coefficient $v_{1}(\eta)$}

Directed flow coefficient $v_{1}(\eta)$ reflects the collective sideward deflection of particles. Here $v_{1}(\eta)$ is calculated via integration as follows,

$$
v_{1}(\eta)=\left\langle\cos \left(\phi-\Psi_{1}\right)\right\rangle=\frac{\int \cos \left(\phi-\Psi_{1}\right) \frac{d N}{d \eta d \phi} d \phi}{\int \frac{d N}{d \eta d \phi} d \phi},
$$

where $\Psi_{1}$ is the first-order event plane of the collision [15].

\section{NUMERICAL RESULTS}

In this section, numerical results from ideal CLVisc hydrodynamic simulation with experimental data for RHIC and the LHC energies are presented.

\section{A. $\mathrm{Cu}+\mathrm{Cu}$ and $\mathrm{Au}+\mathrm{Au} \sqrt{s_{N N}}=200 \mathrm{GeV}$ collisions}

Figure 3 shows a comparison of charged hadron pseudorapidity distributions $d N / d \eta$ between our model and the 

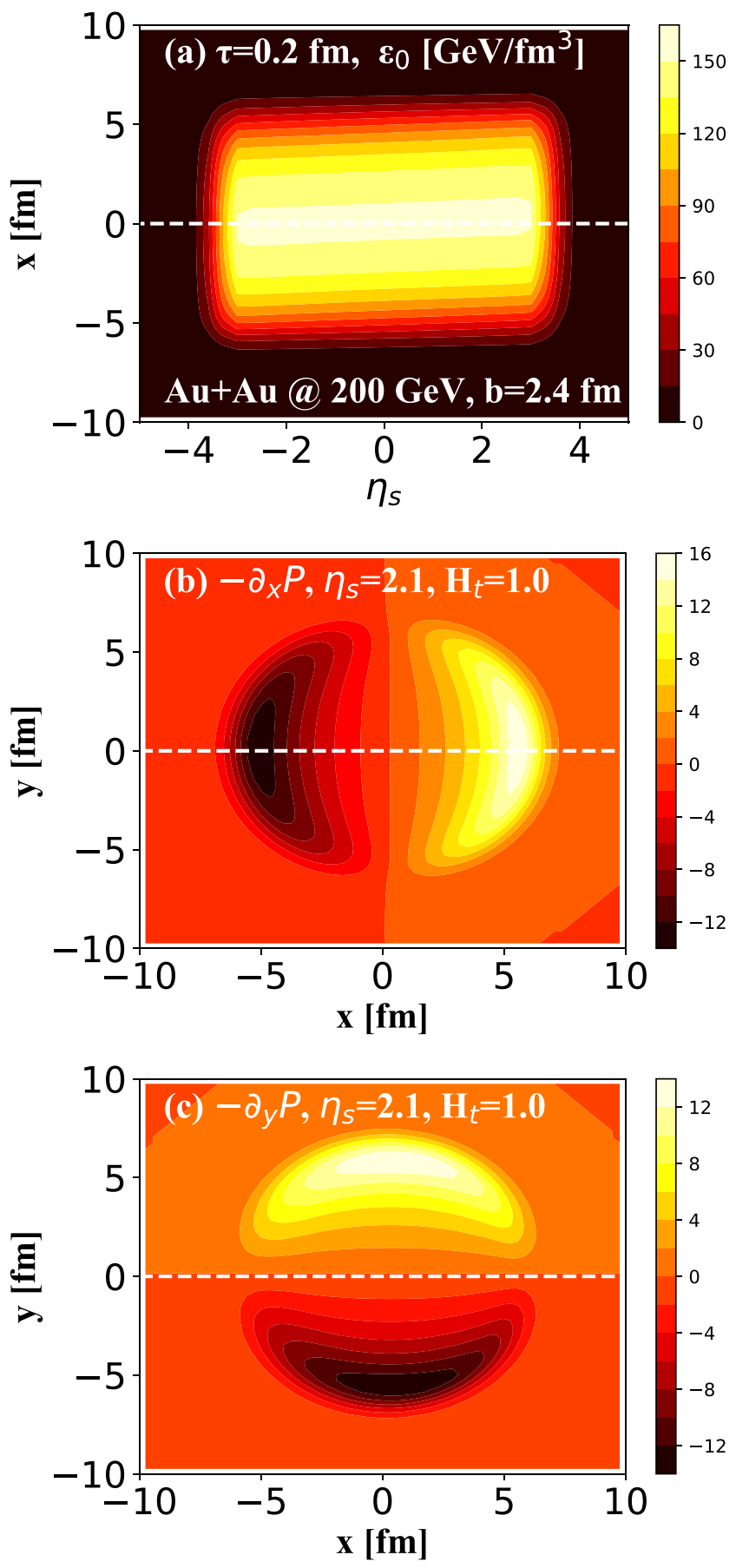

FIG. 1. Color contours show the initial condition at $\tau=0.2 \mathrm{fm}$ in $0 \%-5 \% \mathrm{Au}+\mathrm{Au}$ collisions at $\sqrt{s_{N N}}=200 \mathrm{GeV}$. (top panel) The initial energy density profile on the $\eta_{s}-x$ plane. (middle and bottom panels) The magnitude of pressure gradients $-\partial_{x} P$ and $-\partial_{y} P$ at $\tau_{0}=$ $0.2 \mathrm{fm}$ and $\eta_{s}=2.1$. The impact parameter $b=2.4 \mathrm{fm}$ is consistent with the centrality class $0 \%-5 \%$.

PHOBOS measurement [53] in the most-central centrality $0 \%-6 \% 3 \mathrm{Au}+\mathrm{Au}$ and $\mathrm{Cu}+\mathrm{Cu}$ collisions at $\sqrt{s_{N N}}=200 \mathrm{GeV}$.

${ }^{3}$ If one modifies the impact parameter $b$ and makes it consistent with the specified centrality class, then the model can fitting the multiplicity distribution $d N / d \eta$ well.
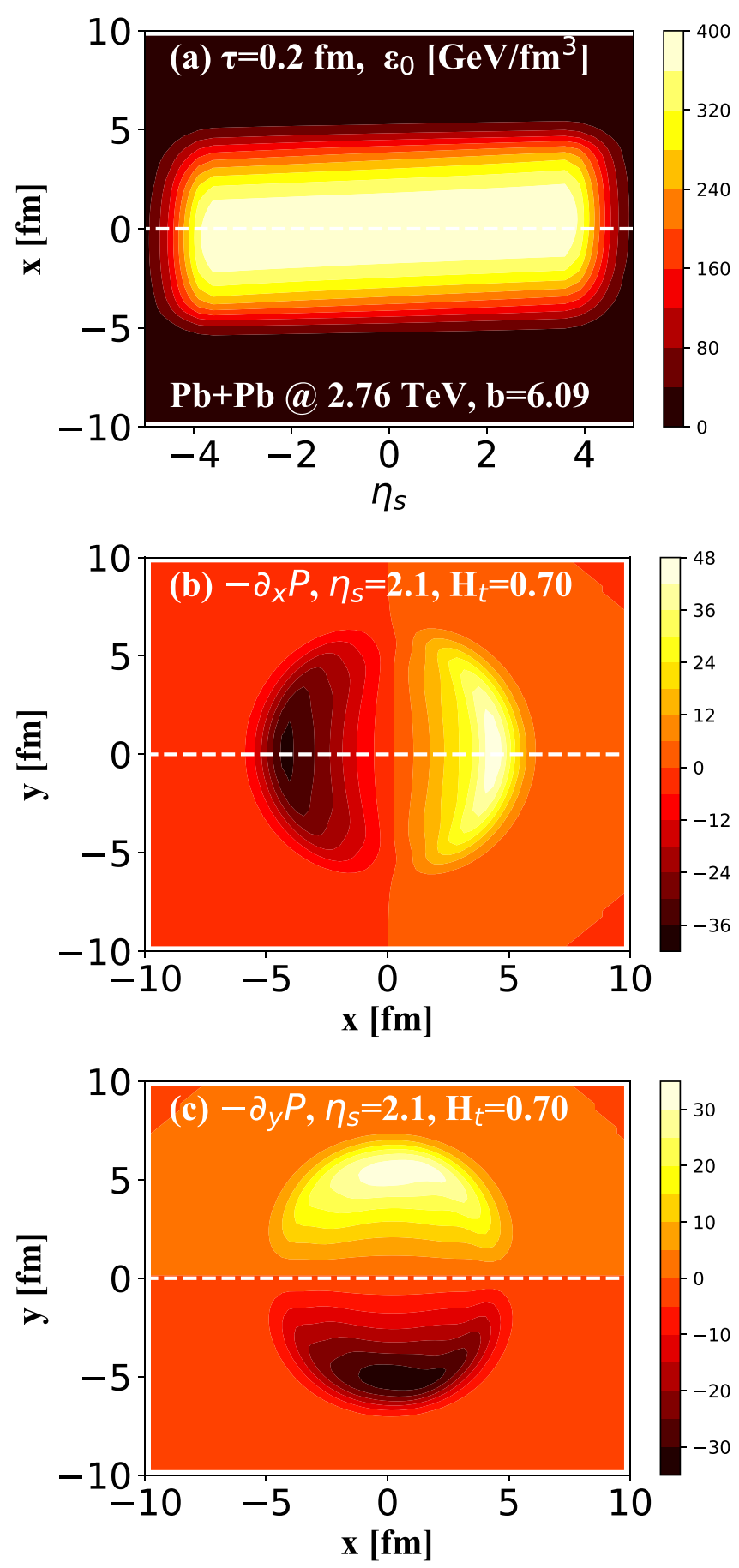

FIG. 2. Color contour plot for initial condition at $\tau=0.2 \mathrm{fm}$ in $10 \%-20 \% \mathrm{~Pb}+\mathrm{Pb}$ collisions at $\sqrt{s_{N N}}=2.76 \mathrm{TeV}$. (top panel) the initial energy density profile on the $\eta_{s^{-}} x$ plane (at $y=0.0 \mathrm{fm}$ ). (middle and bottom panels) The magnitude of pressure gradients with $H_{t}=0.7$ at forward rapidity at $\tau_{0}=0.2 \mathrm{fm}$ and $\eta_{s}=2.1$. The impact parameter $b=6.09 \mathrm{fm}$ is consistent with the centrality class $10 \%-20 \%$.

We find that the $d N / d \eta$ obtained from the ideal CLVisc with modified initial conditions under three different settings of $H_{t}$ are almost indistinguishable on the plot.

Figure 4 shows elliptic flow coefficients $v_{2}\left(p_{\mathrm{T}}\right)$ of the charged particle in only 10\%-20\% $\mathrm{Au}+\mathrm{Au}$ (top panel) and 

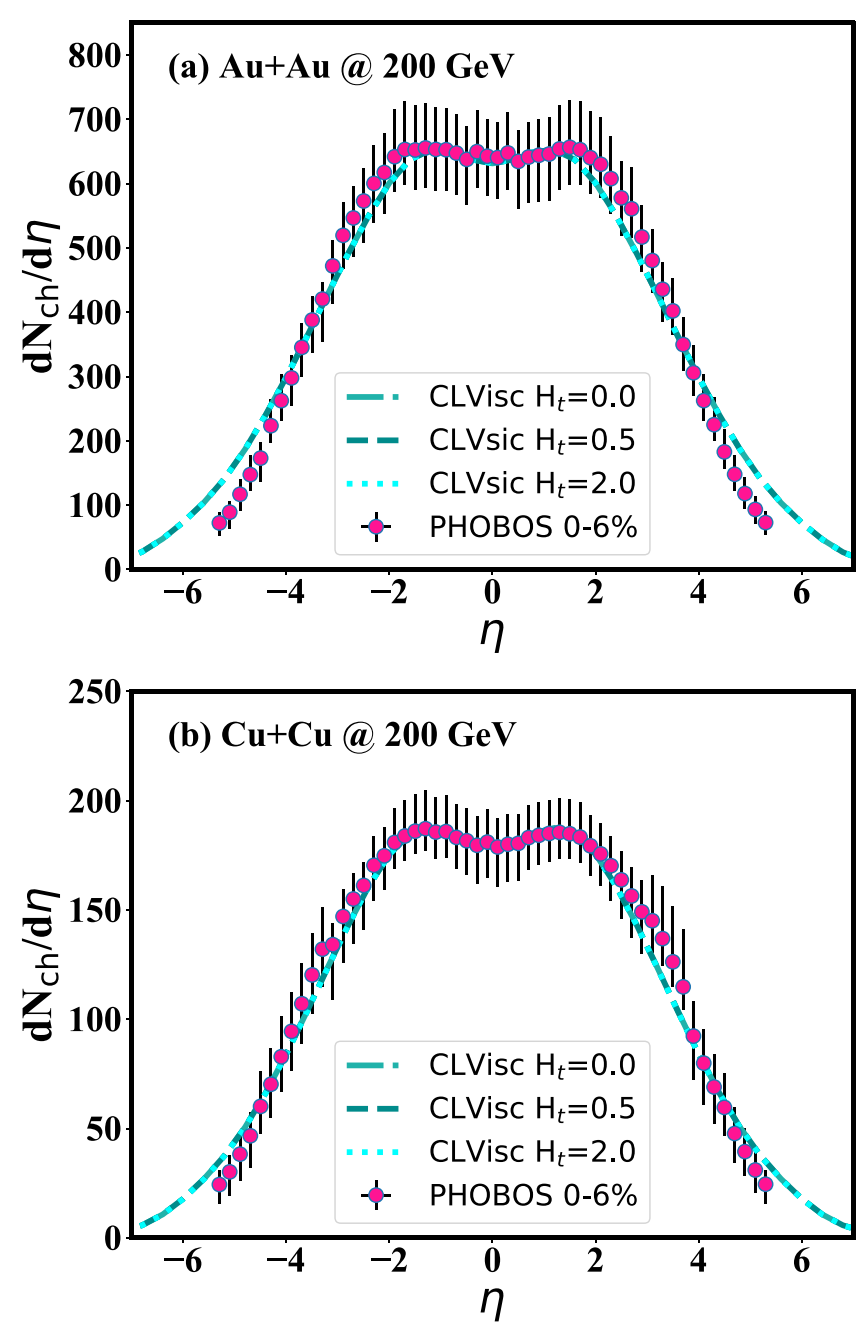

FIG. 3. The charged particle pseudorapidity distribution from CLVsic for (a) $\mathrm{Au}+\mathrm{Au} \sqrt{s_{N N}}=200 \mathrm{GeV}$ collisions and (b) $\mathrm{Cu}+$ $\mathrm{Cu} \sqrt{s_{N N}}=200 \mathrm{GeV}$ collisions in comparison with the experiment data from PHOBOS Collaboration at top RHIC energy [53] with three different settings of $H_{t}$ in the centrality class $0 \%-6 \%$.

$\mathrm{Cu}+\mathrm{Cu}$ (bottom panel) collisions at $\sqrt{s_{N N}}=200 \mathrm{GeV} .^{4}$ Our hydrodynamic simulations with modified initial conditions give a same distribution of $v_{2}\left(p_{\mathrm{T}}\right)$ under different $H_{t}$ compared with the STAR measurement [56].

Our theoretical calculations of pseudorapidity distribution $d N / d \eta$ and elliptic flow $v_{2}\left(p_{\mathrm{T}}\right)$ for different settings of tilted parameter $H_{t}$ show that different initial longitudinal tilted fireball almost does not affect the pseudorapidity distribution and the elliptic flow coefficient $v_{2}\left(p_{T}\right)$.

\footnotetext{
${ }^{4}$ Please notice that our model following those assumptions: (1) modified optical Glauber model, which miss event-by-event eccentricity fluctuations; (2) the dissipative effect is not included during the hydro expansion; (3) the freeze-out temperature $T_{f r z}=137 \mathrm{MeV}$. Above assumptions makes our model sightly overestimate the elliptic flow coefficient $v_{2}\left(p_{\mathrm{T}}\right)$ and $v_{2}(\eta)$ in several centrality classes at the current stage $[17,54,55]$. We will focus on event-by-event dissipative hydrodynamic simulations in future work.
}
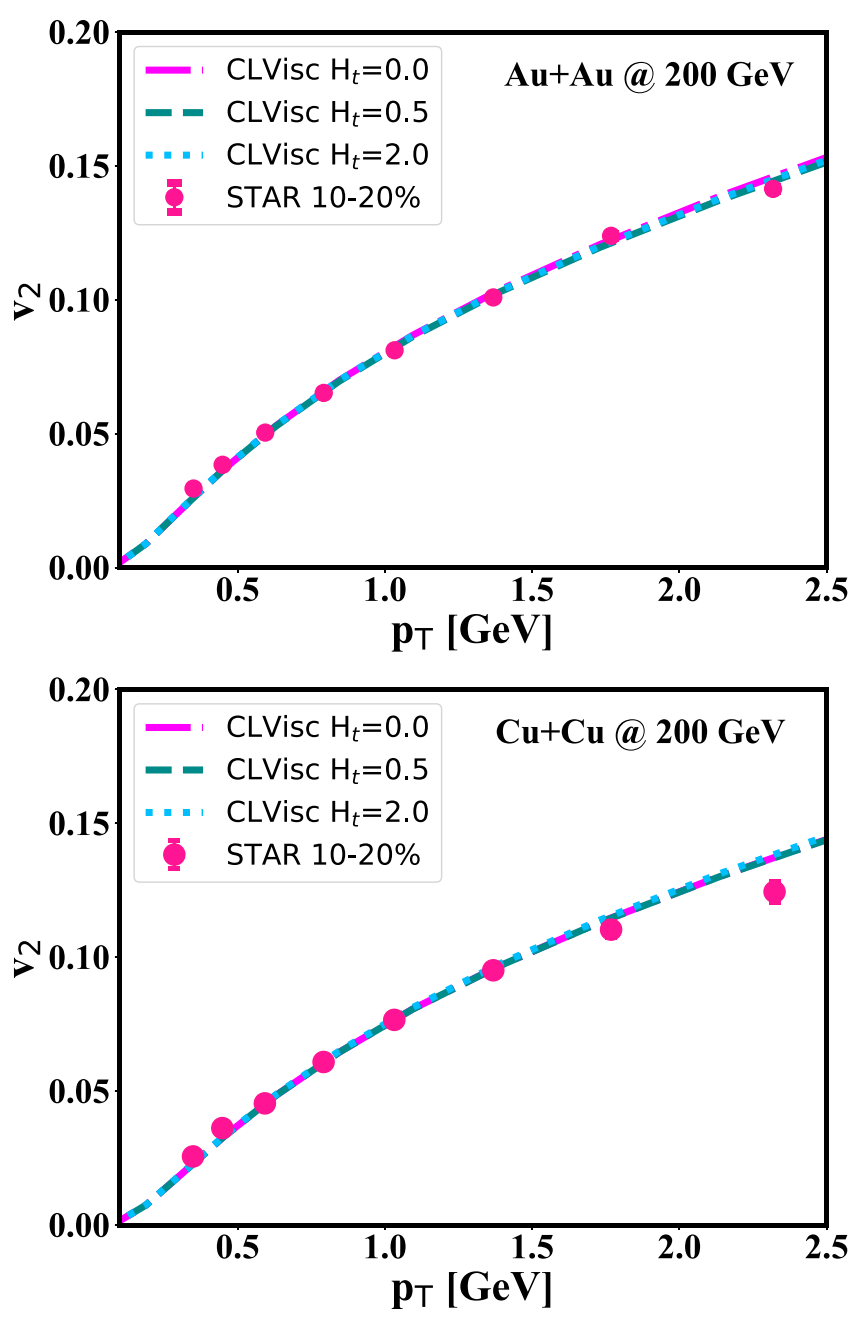

FIG. 4. Elliptic flow coefficients $v_{2}\left(p_{\mathrm{T}}\right)$ of charged particles at RHIC energy for the centrality class $10 \%-20 \%$. (top panel) $\mathrm{Au}+\mathrm{Au}$ $\sqrt{s_{N N}}=200 \mathrm{GeV}$ collisions. (bottom panel) $\mathrm{Cu}+\mathrm{Cu} \sqrt{s_{N N}}=200$ $\mathrm{GeV}$ collisions. The experimental data come from the STAR Collaboration [56].

However, a nonzero directed flow $v_{1}$ can be generated in the hydrodynamic simulation with modified initial conditions. Figure 5 shows the result for the directed flow coefficient $v_{1}(\eta)$ of charged particles emitted after a hydrodynamic evolution. The dashed (-dotted) curves are the results for $\mathrm{Au}+$ $\mathrm{Au}$ and $\mathrm{Cu}+\mathrm{Cu} \sqrt{s_{N N}}=200 \mathrm{GeV}$ collisions. One finds the experimental data in the centrality classes $0 \%-5 \%$ and $5 \%-$ $40 \%$ are reproduced well in a large pseudorapidity region. For the peripheral collisions (in the centrality bin 30\%-60\%), our model overestimates the directed flow coefficient $v_{1}$ at large rapidity (fragmentation region). The directed flow in such a region maybe has different origins, such as the baryon stopping effect and fluctuations $[13,15]$.

\section{B. $\mathrm{Pb}+\mathrm{Pb} \sqrt{{s_{N N}}_{1}}=2.76 \mathrm{TeV}$ and $5.02 \mathrm{TeV}$ collisions}

Figure 6 shows only the most-central pseudorapidity distribution (0\%-5\%) for charged particles from the ideal CLVisc is in comparison with experimental data from the ALICE 

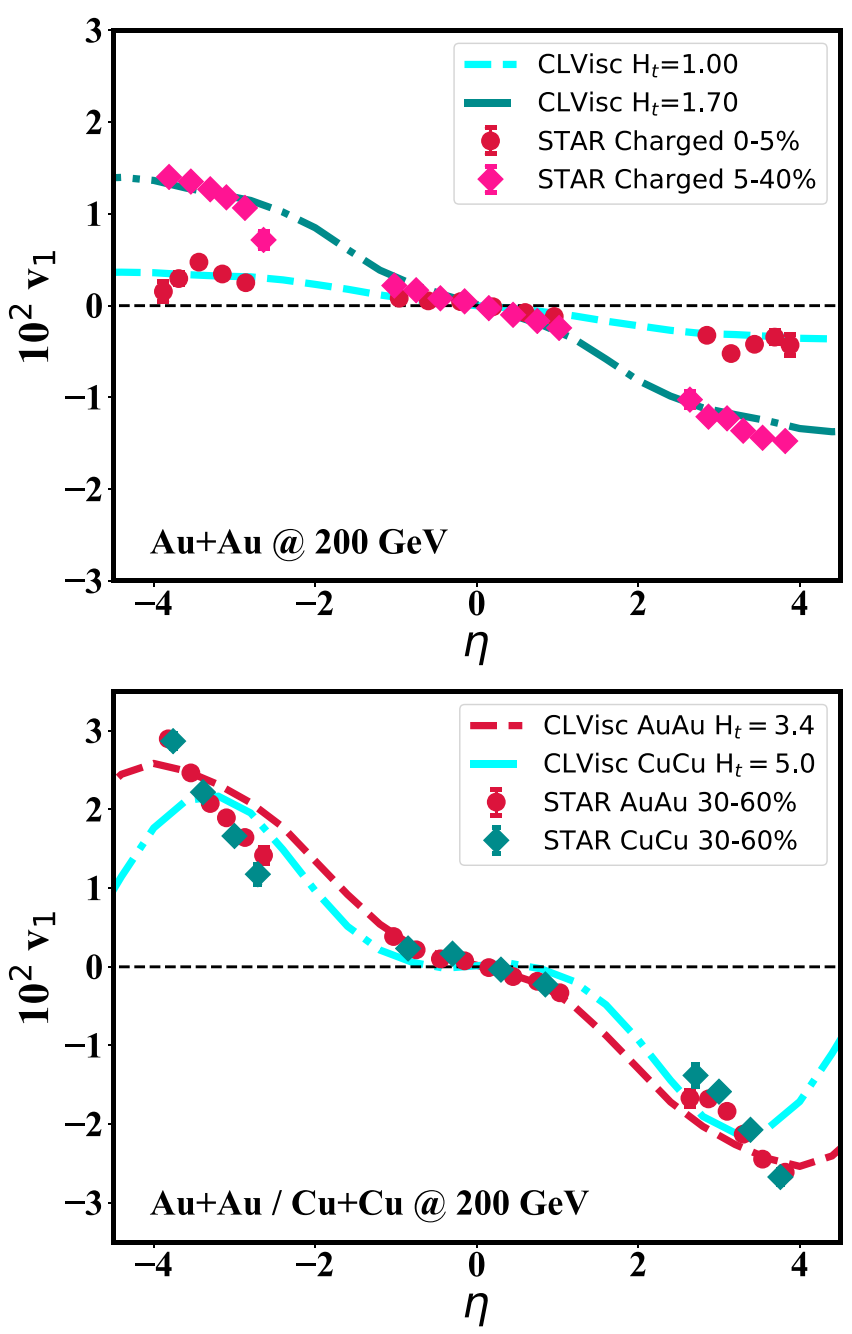

FIG. 5. Directed flow coefficients $v_{1}(\eta)$ of charged particle versus pseudorapidity from CLVsic (colored curves) for $\mathrm{Au}+\mathrm{Au}$ and $\mathrm{Cu}+\mathrm{Cu} \sqrt{s_{N N}}=200 \mathrm{GeV}$ collisions in comparison with the experiment data from the STAR Collaboration at RHIC energy (solid points) [8]. (top panel) Results for centrality classes $0 \%-5 \%$, $5 \%-40 \%$. (bottom panel) Results for centrality class 30\%-60\%.

Collaboration $[57,58]$. The hydrodynamic simulation with different longitudinal tilted initial condition $\left(H_{t}=0.0,0.5\right.$, 2.0) gives almost the same charged multiplicity distribution $d N / d \eta$ for the most-central collisions.

In Fig. 7 we plot the elliptic flow $v_{2}\left(p_{T}\right)$ only for $\mathrm{Pb}+\mathrm{Pb}$ $\sqrt{s_{N N}}=2.76 \mathrm{TeV}$ collisions in the centrality bin $10 \%-15 \%$ (top panel) and $\mathrm{Pb}+\mathrm{Pb} \sqrt{s_{N N}}=5.02 \mathrm{TeV}$ collision (bottom panel) in the centrality bin $10 \%-20 \%$. We find that the elliptic flow $v_{2}\left(p_{T}\right)$ is indistinguishable for different $H_{t}$ (dashed and dashed-dotted curves). The experimental data are from the CMS Collaboration [59] and ATLAS Collaboration [60].

In Fig. 8 we plot the pseudorapidity dependence of the directed flow $v_{1}(\eta)$ for pions $\left(\pi^{+}\right)$. Figure 8 (top panel) shows the results for $\mathrm{Pb}+\mathrm{Pb} \sqrt{s_{N N}}=2.76 \mathrm{TeV}$ in the centrality classes $10 \%-20 \%$ and $30 \%-40 \%$. We see that hydrodynamic simulation with modified initial conditions reproduce the experimental data from ALICE Collaboration [61] in the
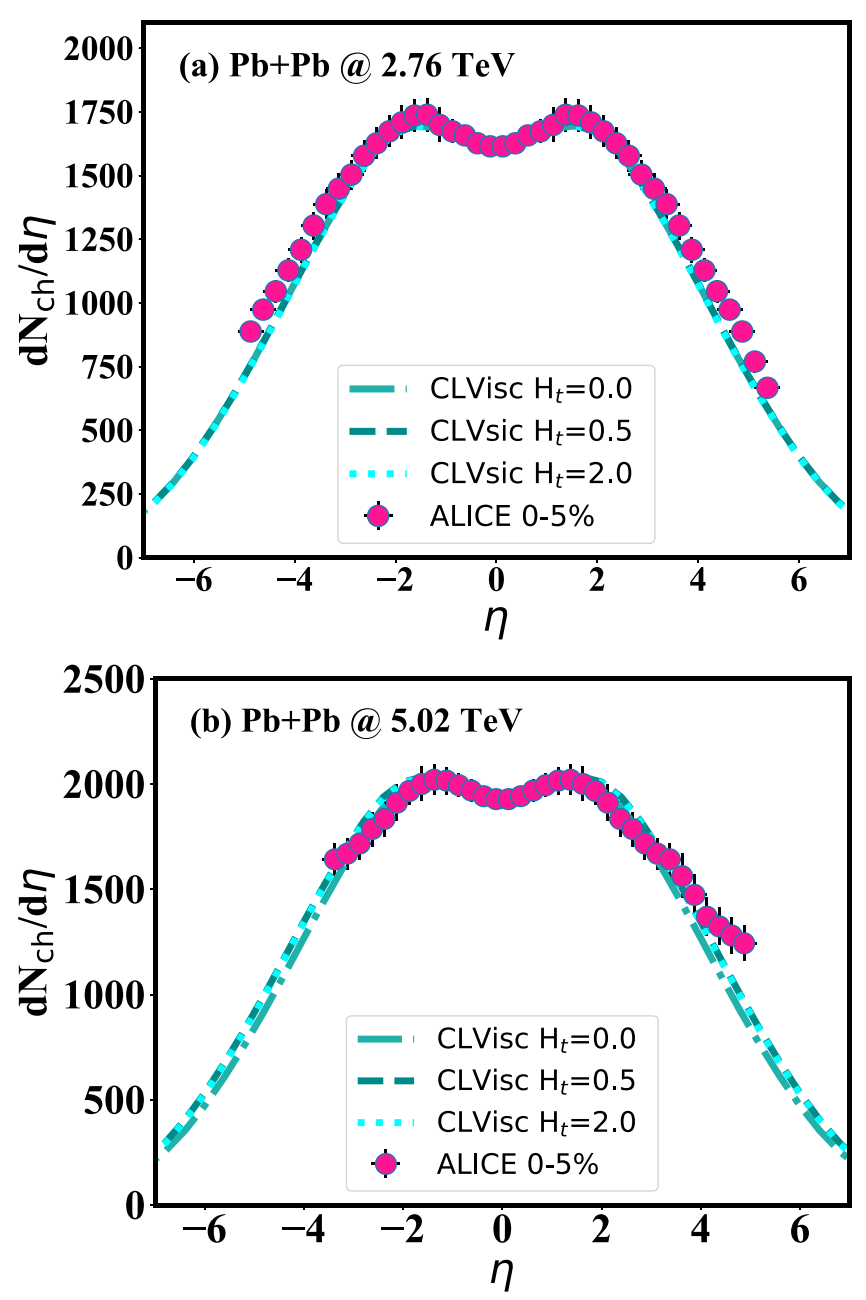

FIG. 6. The charged particle pseudorapidity distribution from CLVsic (colored curves) for $\mathrm{Pb}+\mathrm{Pb} \sqrt{s_{N N}}=2.76 \mathrm{TeV}$ [57] and $\sqrt{s_{N N}}=5.02 \mathrm{TeV}$ [58] collisions at the LHC energy in comparison with the experiment data from ALICE Collaboration (solid points).

centrality bin $10 \%-20 \%$ with tilted parameter $H_{t}=0.7$ and $30 \%-40 \%$ with $H_{t}=0.8$.

Figure 8 (bottom panel) shows the result for $\mathrm{Pb}+\mathrm{Pb}$ $\sqrt{s_{N N}}=5.02 \mathrm{TeV}$ in the centrality class $5 \%-40 \%$. We see that the ALICE Collaboration [11] points for the centrality class $5 \%-40 \%$ are for positive charged particles, and results for the pion $\left(\pi^{+}\right)$directed flow from the CLVisc simulation agree with the experimental data to a reasonable level. This is because (a) a distinction for the whole positive and negative particle yields needs a proper theory, which has not been developed totally in the current stage; (b) $\pi^{+}$plays a vital role for the yield of the total positive charge particles and mainly comes from the hydrodynamic evolution [57,58].

To get information about how the longitudinal tilted structure introduced in Eq. (5) is related to the final azimuthal asymmetry measured by the directed flow, we plot the $H_{t}$ dependence of the directed flow of charged particles in Fig. 9 for $\mathrm{Pb}+\mathrm{Pb} \sqrt{s_{N N}}=2.76 \mathrm{TeV}$ collisions in the centrality bin $10 \%-20 \%$. We find a larger value of $H_{t}$ corresponds to a more longitudinal tilted fireball and leads to a larger value of the 

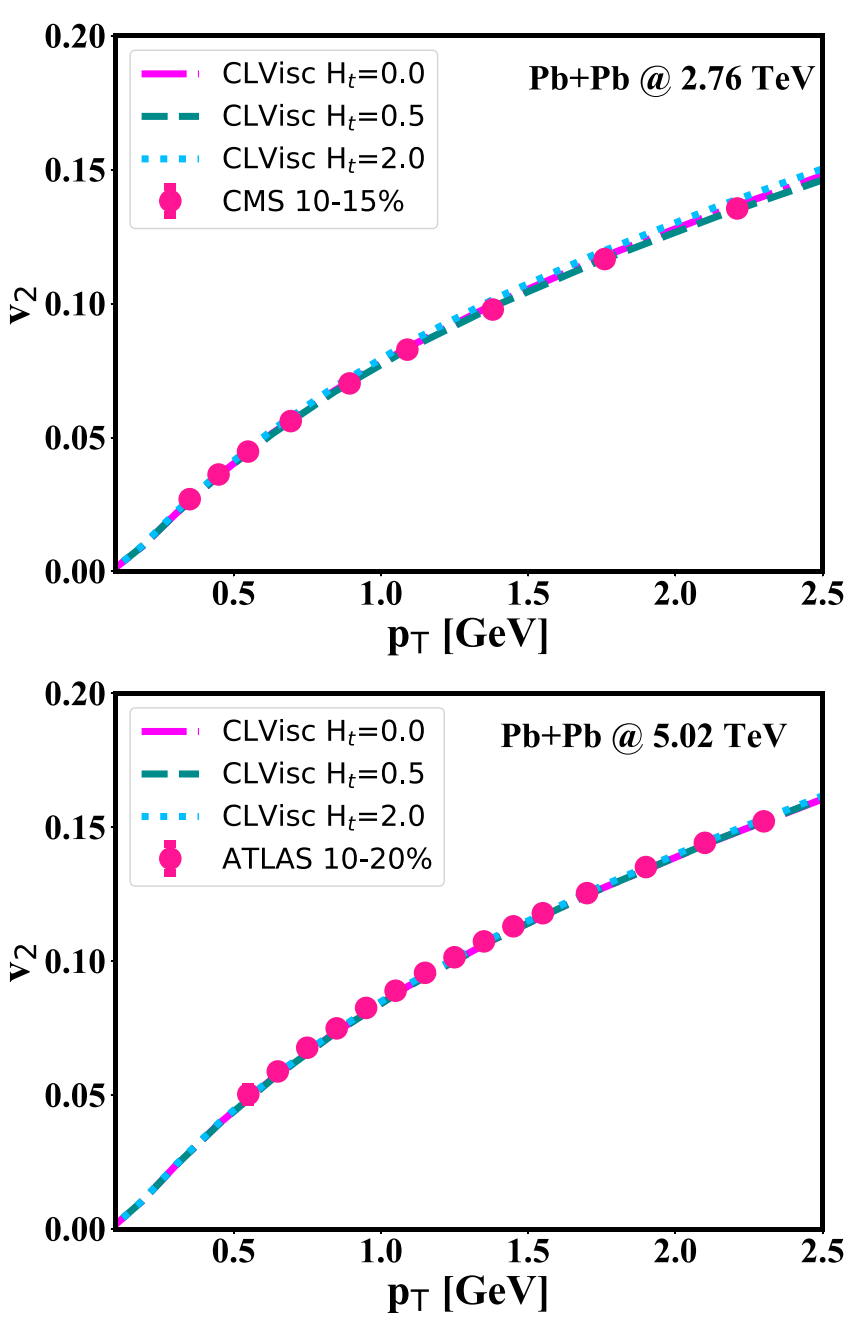

FIG. 7. Elliptic flow coefficients $v_{2}\left(p_{T}\right)$ for $\mathrm{Pb}+\mathrm{Pb}$ collisions at the LHC energy. (top panel) Result in $10 \%-15 \% \mathrm{~Pb}+\mathrm{Pb}$ collisions at $\sqrt{s_{N N}}=2.76 \mathrm{TeV}$, the experimental data come form the CMS Collaboration [59]. (bottom panel) Results for $\mathrm{Pb}+\mathrm{Pb} \sqrt{s_{N N}}=$ $5.02 \mathrm{TeV}$ collisions for the centrality class $10 \%-20 \%$, the experimental data come from the ATLAS Collaboration [60].

directed flow coefficient at large rapidity. The value of $H_{t}$ extracted from the STAR and ALICE data show that (a) the initial spatial pressure gradient asymmetry in the transverse plane of bulk medium at RHIC energy is larger than at the LHC energy at the initial proper time; (b) the larger the impact parameter $b$, the larger the magnitude of the asymmetry of the initial pressure gradient in the transverse plane at the initial stage.

\section{SUMMARY}

In this work, following the previous work Refs. [15,21,27,40], an alternative parametrization to construct longitudinal tilted initial condition based on the Glauber model is presented. A data-driven phenomenological rapidity-dependent weight function [Eq. (5)] is obtained to generate the initial tilting longitudinal expansion of the strongly coupled QCD matter, and the results show that such a
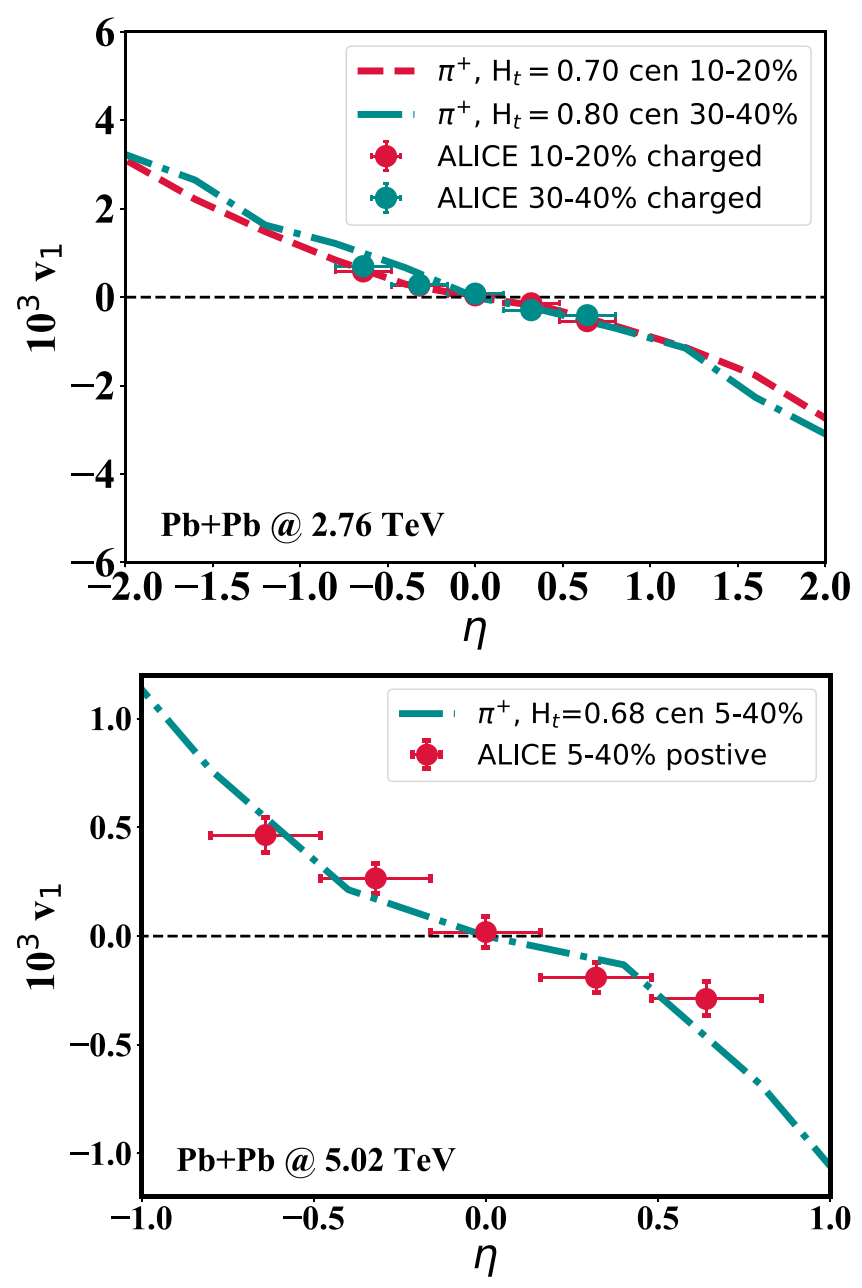

FIG. 8. Directed flow coefficients $v_{1}(\eta)$ of $\pi^{+}$versus pseudorapidity from CLVsic (colored curves) for $\mathrm{Pb}+\mathrm{Pb}$ collisions at the LHC energy in comparison with the experiment data from the ALICE Collaboration (solid points) [11,61]. (top panel) Results for $\mathrm{Pb}+\mathrm{Pb}$ $\sqrt{s_{N N}}=2.76 \mathrm{TeV}$ collision at centrality class $10 \%-20 \%, 30 \%-40 \%$. (bottom panel) Results for $\mathrm{Pb}+\mathrm{Pb} \sqrt{s_{N N}}=5.02 \mathrm{TeV}$ collision at centrality class $5 \%-40 \%$.

tilting source leads to a magnitude asymmetry of the pressure gradient along the $x$ direction and longitudinal $\eta_{s}$ direction [15]. The existence of such asymmetries push the light quarks and gluons toward the $x>0$ (and $x<0$ ) direction at different rapidities, which gives a nonzero directed flow coefficient.

The pseudorapidity distribution $d N / d \eta$ and the elliptic flow coefficient $v_{2}\left(p_{T}\right)$ of charged particles are presented for $\mathrm{Cu}+\mathrm{Cu}, \mathrm{Au}+\mathrm{Au}$, and $\mathrm{Pb}+\mathrm{Pb}$ collisions for serval longitudinal tilted parameters $H_{t}$. The longitudinal tilted structure in hot-QCD matter affect both the multiplicity density distribution and elliptic flow coefficient weakly and almost negligible, consistent with previous studies $[15,40]$.

Our modified initial conditions with the ideal CLVisc hydrodynamic simulation is used to fit the directed flow coefficient $v_{1}(\eta)$ of charged particles and pions measured by the STAR Collaboration and the ALICE Collaboration. We find that the directed flow coefficient is generated at a very early stage in the evolution and is given by the initial tilted source. 


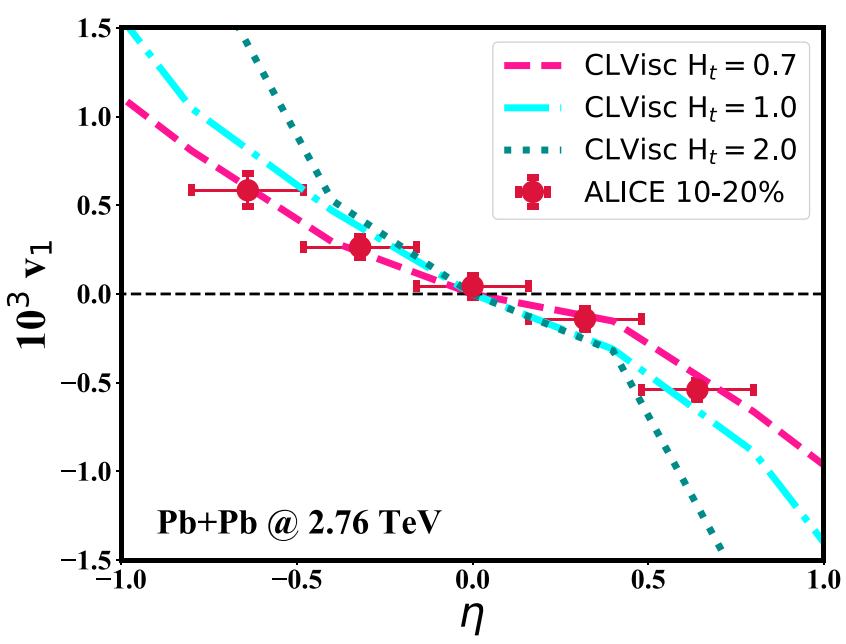

FIG. 9. Directed flow coefficients $v_{1}(\eta)$ of charged particles versus pseudorapidity from CLVsic (colored curves) for $\mathrm{Pb}+\mathrm{Pb}$ collisions at the LHC energy in comparison with the experiment data from the ALICE Collaboration with a different model parameter $H_{t}$.

The directed flow coefficient is decreased with the center-ofmass energy $\sqrt{s_{N N}}$, the reasons are (a) with increasing range of longitudinal rapidity the imbalance between the thickness function $T_{1}$ and $T_{2}$ goes down; (b) the contribution of binary collisions to the Glauber model is believed to increase with the $\sqrt{s_{N N}}[27,40]$.

We also remark that, besides the initial fireball spatial asymmetry contributing to the directed flow, the following aspects are also important and can be extended to future work.

(1) In real heavy ion collisions, the fluctuations of the initial energy density also contribute to the local pressure gradient asymmetry, e.g., $\mathrm{T}_{\mathrm{R}}$ ENTo-3D initial condition model [62]. It is possible that the dynamical fluctuations or the initial flow in the $z$ direction can generate a large pressure gradient asymmetry for both angle and magnitude [15-17,28,62].

(2) During the hydrodynamic expansion, the viscosity corrections reduce the longitudinal pressure and increase the transverse pressure. As a result, a smaller directed flow coefficient is observed after a viscous hydrodynamic simulation. This means that the ideal hydrodynamics is a suitable tool for the study of the presence of directed flow in heavy ion collisions [40]. The expansion of the strongly coupled matter is affected by the shear viscosity and bulk viscosity in both the transverse plane and the longitudinal direction. The observables such as the elliptic flow and $p_{T}$ spectra are more sensitive to the viscosity effect according to viscous hydrodynamics calculations $[31,54,55,63,64]$. Moreover, the $v_{2}(\eta)$ coefficient is sightly overestimated in our model as the lack of the shear viscosity effect $[31,40]$. Because $v_{2}(\eta)$ places severe constraints to the initial shape of the fireball away from midrapidity, and thus to the parametrization of the initial state $[31,54,55]$. In the future study, viscous correction will be included and the $v_{2}(\eta)$ coefficient will be used to constrain our initial condition.

(3) For noncentral $A+A$ collisions, an extremely strong magnetic field is created by the colliding charged beams moving at relativistic speed (almost $10^{16}-10^{20}$ Gauss) [65-68]. Due to the expansion along the beam axis, the Lorentz force is directed along the negative- $x$ direction in the forward-rapidity region for positively charged quarks, which generates a directed transverse flow. In addition to the above Hall effect, the time dependence of the magnetic field generates an electric field due to the Faraday effect. The induced Faraday current provides a no-zero finite drift velocity in the transverse plane due to the magnetic field. Lots of work has investigated the contribution of the combination of the above two effects on the directed flow coefficient, such as the MHD [27] and decoupled hydro-magnetic frame [22,24]. And one has found that the contribution of the magnetic-field effect on the soft hadron directed flow coefficient is less than $5 \times 10^{-4}$ at large rapidity, which is almost $80-100$ times smaller than the contribution from the tilted initial conditions [27].

(4) Taking into account the asymmetry between forward and backward moving participants, the noncentral heavy ion collisions produce not only strong angular momentum and strong magnetic field but also global and local vorticity and hyperon polarization [28,32].

(5) The directed flow of heavy hadrons could be a great probe to investigate the initial pressure gradient asymmetry of bulk matter $[16,23]$. Vice versa, such kind of tilted medium could be the background of heavy quark propagation, and its effect on open heavy flavor production $v_{1}$ and $R_{A A}$ might be interesting problems [21,69-72]. Furthermore, $D^{0}$ directed flow found at both STAR and ALICE still contains large statistical uncertainly and systemic uncertainly, one suggests that hydrodynamic + transport model together may put more constraints on the directed flow for better understanding of the initial stage in heavy ion collisions [26].

(6) We also need to take into account the hadronic cascade. For a proper comparison with more experimental data, one should include such interactions with the addition of hadronic transport models such as UrQMD $[73,74]$ and SMASH $[75,76]$.

(7) Recently, a large number of studies used the parametrizations for the longitudinal structure of the fireball in Ref. [15] to investigate the directed flow coefficient of heavy mesons [19-21,28]. Collisiongeometry-based 3D initial conditions with hydrodynamic simulations from the group of Shen et al. $[17,18]$ also described the directed flow coefficient of $\pi^{+}$at RHIC energies well. It will be interesting to see which one of the models can lead to the reproduction of the experimental data with the least amount of tweaking of the parameters.

These important aspects will be studied in the future. 


\section{ACKNOWLEDGMENTS}

The authors thank Xin-Nian Wang for helpful comments and providing the GPU computing platform at the initial stage of this study. Z.-F.J. would like to thank Xiangyu Wu, ShanShan Cao, Chun Shen, Chi Ding, Zhong Yang, and LongGang Pang for helpful discussion. The authors would like to thank the anonymous reviewers for their helpful remarks. This research was supported by the NSFC of China with Project No. 11935007, the Xiaogan Natural Science Founda- tion under Grant No. XGKJ2021010016, and the Department of Education of Hubei Province of China with Young Talents Project No. Q20212703. Computational resources have been provided by the Center of Scientific Computing at the Department of Physics and Electronic-Information Engineering, Hubei Engineering University. The numerical simulations have been preformed partly at the GPU cluster in the Nuclear Science Computing Center at Central China Normal University $\left(\mathrm{NSC}^{3}\right)$.
[1] S. A. Bass, M. Gyulassy, H. Stoecker, and W. Greiner, Signatures of quark gluon plasma formation in high-energy heavy ion collisions: A critical review, J. Phys. G 25, R1 (1999).

[2] M. Gyulassy and L. McLerran, New forms of QCD matter discovered at RHIC, Nucl. Phys. A 750, 30 (2005).

[3] E. Shuryak, Why does the quark gluon plasma at RHIC behave as a nearly ideal fluid? Prog. Part. Nucl. Phys. 53, 273 (2004).

[4] U. Heinz and R. Snellings, Collective flow and viscosity in relativistic heavy-ion collisions, Annu. Rev. Nucl. Part. Sci. 63 , 123 (2013).

[5] J.-Y. Ollitrault, Anisotropy as a signature of transverse collective flow, Phys. Rev. D 46, 229 (1992).

[6] S. Voloshin and Y. Zhang, Flow study in relativistic nuclear collisions by Fourier expansion of azimuthal particle distributions, Z. Phys. C: Part. Fields 70, 665 (1996).

[7] B. B. Back et al., The PHOBOS perspective on discoveries at RHIC, Nucl. Phys. A 757, 28 (2005).

[8] B. I. Abelev et al., System-Size Independence of Directed Flow at the Relativistic Heavy-Ion Collider, Phys. Rev. Lett. 101, 252301 (2008).

[9] L. Adamczyk et al., Beam-Energy Dependence of the Directed Flow of Protons, Antiprotons, and Pions in $\mathrm{Au}+\mathrm{Au}$ Collisions, Phys. Rev. Lett. 112, 162301 (2014).

[10] J. Adam et al., First Observation of the Directed Flow of $D^{0}$ and $\overline{D^{0}}$ in $\mathrm{Au}+\mathrm{Au}$ Collisions at $\sqrt{s_{\mathrm{NN}}}=200 \mathrm{GeV}$, Phys. Rev. Lett. 123, 162301 (2019).

[11] S. Acharya et al., Probing the Effects of Strong Electromagnetic Fields with Charge-Dependent Directed Flow in $\mathrm{Pb}-\mathrm{Pb}$ Collisions at the LHC, Phys. Rev. Lett. 125, 022301 (2020).

[12] L. Adamczyk et al., Beam-Energy Dependence of Directed Flow of $\Lambda, \bar{\Lambda}, K^{ \pm}, K_{s}^{0}$, and $\phi$ in $\mathrm{Au}+\mathrm{Au}$ Collisions, Phys. Rev. Lett. 120, 062301 (2018).

[13] H. Petersen, Qingfeng Li, Xianglei Zhu, and M. Bleicher, Directed and elliptic flow in heavy ion collisions at GSI-FAIR and CERN-SPS, Phys. Rev. C 74, 064908 (2006).

[14] A. Adil and M. Gyulassy, 3D jet tomography of twisted strongly coupled quark gluon plasmas, Phys. Rev. C 72, 034907 (2005).

[15] P. Bozek and I. Wyskiel, Directed flow in ultrarelativistic heavyion collisions, Phys. Rev. C 81, 054902 (2010).

[16] B. Chen, M. Hu, H. Zhang, and J. Zhao, Probe the tilted quarkgluon plasma with charmonium directed flow, Phys. Lett. B 802, 135271 (2020).

[17] C. Shen and S. Alzhrani, Collision-geometry-based 3D initial condition for relativistic heavy-ion collisions, Phys. Rev. C 102, 014909 (2020).

[18] S. Ryu, V. Jupic, and C. Shen, Probing early-time longitudinal dynamics with the $\Lambda$ hyperon's spin polarization in relativistic heavy-ion collisions, Phys. Rev. C 104, 054908 (2021).
[19] S. Chatterjee and P. Bożek, Large Directed Flow of Open Charm Mesons Probes the Three-Dimensional Distribution of Matter in Heavy Ion Collisions, Phys. Rev. Lett. 120, 192301 (2018)

[20] S. Chatterjee and P. Bozek, Interplay of drag by hot matter and electromagnetic force on the directed flow of heavy quarks, Phys. Lett. B 798, 134955 (2019).

[21] A. Beraudo, A. De Pace, M. Monteno, M. Nardi, and F. Prino, Rapidity dependence of heavy-flavour production in heavy-ion collisions within a full $3+1$ transport approach: Quenching, elliptic and directed flow, J. High Energy Phys. 05 (2021) 279.

[22] U. Gursoy, D. E. Kharzeev, and K. Rajagopal, Magnetohydrodynamics, charged currents and directed flow in heavy ion collisions, Phys. Rev. C 89, 054905 (2014).

[23] Santosh K. Das, S. Plumari, S. Chatterjee, J. Alam, F. Scardina, and V. Greco, Directed flow of charm quarks as a witness of the initial strong magnetic field in ultra-relativistic heavy ion collisions, Phys. Lett. B 768, 260 (2017).

[24] U. Gürsoy, D. E. Kharzeev, E. Marcus, K. Rajagopal, and C. Shen, Charge-dependent flow induced by magnetic and electric fields in heavy ion collisions, Phys. Rev. C 98, 055201 (2018).

[25] Y. Sun, S. Plumari, and V. Greco, Probing the electromagnetic fields in ultrarelativistic collisions with leptons from $Z^{0}$ decay and charmed mesons, Phys. Lett. B 816, 136271 (2021).

[26] A. Dubla, U. Gürsoy, and R. Snellings, Charge-dependent flow as evidence of strong electromagnetic fields in heavy-ion collisions, Mod. Phys. Lett. A 35, 2050324 (2020).

[27] G. Inghirami, M. Mace, Y. Hirono, L. Del Zanna, D. E. Kharzeev, and M. Bleicher, Magnetic fields in heavy ion collisions: Flow and charge transport, Eur. Phys. J. C 80, 293 (2020).

[28] L. Oliva, S. Plumari, and V. Greco, Directed flow of $D$ mesons at RHIC and LHC: non-perturbative dynamics, longitudinal bulk matter asymmetry and electromagnetic fields, J. High Energy Phys. 05 (2021) 034.

[29] M. Nasim and S. Singha, Directed flow of open charm in $\mathrm{Au}+\mathrm{Au}$ collisions at $\sqrt{s_{N N}}=200 \mathrm{GeV}$ using a quark coalescence model, Phys. Rev. C 97, 064917 (2018).

[30] https://inspirehep.net/authors/1274264.

[31] L.-G. Pang, H. Petersen, and X.-N. Wang, Pseudorapidity distribution and decorrelation of anisotropic flow within the open-computing-language implementation CLVisc hydrodynamics, Phys. Rev. C 97, 064918 (2018).

[32] L.-G. Pang, H. Petersen, Q. Wang, and X.-N. Wang, Vortical Fluid and $\Lambda$ Spin Correlations in High-Energy Heavy-Ion Collisions, Phys. Rev. Lett. 117, 192301 (2016).

[33] L. Adamczyk et al., Global $\Lambda$ hyperon polarization in nuclear collisions: Evidence for the most vortical fluid, Nature (London) 548, 62 (2017). 
[34] L.-G. Pang, G. Endrődi, and H. Petersen, Magnetic-fieldinduced squeezing effect at energies available at the BNL Relativistic Heavy Ion Collider and at the CERN Large Hadron Collider, Phys. Rev. C 93, 044919 (2016).

[35] L.-G. Pang, H. Petersen, G.-Y. Qin, V. Roy, and X.-N. Wang, Decorrelation of anisotropic flow along the longitudinal direction, Eur. Phys. J. A 52, 97 (2016).

[36] X.-Y. Wu, L.-G. Pang, G.-Y. Qin, and X.-N. Wang, Longitudinal fluctuations and decorrelations of anisotropic flows at energies available at the CERN Large Hadron Collider and at the BNL Relativistic Heavy Ion Collider, Phys. Rev. C 98, 024913 (2018).

[37] L.-G. Pang, Kai Zhou, Nan Su, H. Petersen, H. Stöcker, and X.-N. Wang, An equation-of-state-meter of quantum chromodynamics transition from deep learning, Nat. Commun. 9, 210 (2018).

[38] W. Chen, S. Cao, T. Luo, L.-G. Pang, and X.-N. Wang, Effects of jet-induced medium excitation in $\gamma$-hadron correlation in $A+A$ collisions, Phys. Lett. B 777, 86 (2018).

[39] Y. He, L.-G. Pang, and X.-N. Wang, Bayesian Extraction of Jet Energy Loss Distributions in Heavy-ion Collisions, Phys. Rev. Lett. 122, 252302 (2019).

[40] P. Bożek, Flow and interferometry in $3+1$ dimensional viscous hydrodynamics, Phys. Rev. C 85, 034901 (2012).

[41] https://gitlab.com/snowhitiger/PyVisc.

[42] M. L. Miller, K. Reygers, S. J. Sanders, and P. Steinberg, Glauber modeling in high energy nuclear collisions, Annu. Rev. Nucl. Part. Sci. 57, 205 (2007).

[43] C. Loizides, J. Kamin, and D. d'Enterria, Improved Monte Carlo Glauber predictions at present and future nuclear colliders, Phys. Rev. C 97, 054910 (2018); 99, 019901(E) (2019).

[44] Z. F. Jiang, D. She, C. B. Yang, and D. Hou, Perturbation solutions of relativistic viscous hydrodynamics for longitudinally expanding fireballs, Chin. Phys. C 44, 084107 (2020).

[45] Z. F. Jiang, C. B. Yang, C. Ding, and X.-Y. Wu, Pseudo-rapidity distribution from a perturbative solution of viscous hydrodynamics for heavy ion collisions at RHIC and LHC, Chin. Phys. C 42, 123103 (2018).

[46] G. S. Denicol, H. Niemi, E. Molnar, and D. H. Rischke, Derivation of transient relativistic fluid dynamics from the Boltzmann equation, Phys. Rev. D 85, 114047 (2012); 91, 039902 (2015).

[47] P. Romatschke, New developments in relativistic viscous hydrodynamics, Int. J. Mod. Phys. E 19, 1 (2010).

[48] P. Romatschke and U. Romatschke, Relativistic Fluid Dynamics In and Out of Equilibrium, Cambridge Monographs on Mathematical Physics (Cambridge University Press, 2019), Vol. 5.

[49] S. Borsanyi, Z. Fodor, C. Hoelbling, S. D. Katz, S. Krieg, and K. K. Szabo, Full result for the QCD equation of state with 2+1 flavors, Phys. Lett. B 730, 99 (2014).

[50] B. Schenke, Sangyong Jeon, and C. Gale, $(3+1) D$ hydrodynamic simulation of relativistic heavy-ion collisions, Phys. Rev. C 82, 014903 (2010).

[51] Y.-L. Du, K. Zhou, J. Steinheimer, L.-G. Pang, A. Motornenko, H.-S. Zong, X.-N. Wang, and H. Stöcker, Identifying the nature of the QCD transition in relativistic collision of heavy nuclei with deep learning, Eur. Phys. J. C 80, 516 (2020).

[52] F. Cooper and G. Frye, Comment on the single particle distribution in the hydrodynamic and statistical thermodynamic models of multiparticle production, Phys. Rev. D 10, 186 (1974).
[53] B. Alver et al., Phobos results on charged particle multiplicity and pseudorapidity distributions in $\mathrm{Au}+\mathrm{Au}, \mathrm{Cu}+\mathrm{Cu}, d+\mathrm{Au}$, and $p+p$ collisions at ultra-relativistic energies, Phys. Rev. C 83, 024913 (2011).

[54] T. Hirano and K. Tsuda, Collective flow and two-pion correlations from a relativistic hydrodynamic model with early chemical freeze-out, Phys. Rev. C 66, 054905 (2002).

[55] T. Hirano, U. W. Heinz, D. Kharzeev, R. Lacey, and Y. Nara, Hadronic dissipative effects on elliptic flow in ultrarelativistic heavy-ion collisions, Phys. Lett. B 636, 299 (2006).

[56] J. Adams et al., Azimuthal anisotropy in $\mathrm{Au}+\mathrm{Au}$ collisions at $\sqrt{s_{\mathrm{NN}}}=200 \mathrm{GeV}$, Phys. Rev. C 72, 014904 (2005).

[57] J. Adam et al., Centrality evolution of the charged-particle pseudorapidity density over a broad pseudorapidity range in $\mathrm{Pb}-\mathrm{Pb}$ collisions at $\sqrt{s_{\mathrm{NN}}}=2.76 \mathrm{TeV}$, Phys. Lett. B 754, 373 (2016).

[58] J. Adam et al., Centrality dependence of the pseudorapidity density distribution for charged particles in $\mathrm{Pb}-\mathrm{Pb}$ collisions at $\sqrt{s_{\mathrm{NN}}}=5.02 \mathrm{TeV}$, Phys. Lett. B 772, 567 (2017).

[59] S. Chatrchyan et al., Measurement of the elliptic anisotropy of charged particles produced in $\mathrm{Pb}-\mathrm{Pb}$ collisions at $\sqrt{s_{N N}}=$ 2.76TeV, Phys. Rev. C 87, 014902 (2013).

[60] M. Aaboud et al., Measurement of the azimuthal anisotropy of charged particles produced in $\sqrt{s_{\mathrm{NN}}}=5.02 \mathrm{TeV} \mathrm{Pb}+\mathrm{Pb}$ collisions with the ATLAS detector, Eur. Phys. J. C 78, 997 (2018).

[61] B. Abelev et al., Directed Flow of Charged Particles at Midrapidity Relative to the Spectator Plane in $\mathrm{Pb}-\mathrm{Pb}$ Collisions at $\sqrt{s_{N N}}=2.76 \mathrm{TeV}$, Phys. Rev. Lett. 111, 232302 (2013).

[62] W. Ke, J. S. Moreland, J. E. Bernhard, and S. A. Bass, Constraints on rapidity-dependent initial conditions from charged particle pseudorapidity densities and two-particle correlations, Phys. Rev. C 96, 044912 (2017).

[63] H. Song and U. W. Heinz, Suppression of elliptic flow in a minimally viscous quark-gluon plasma, Phys. Lett. B 658, 279 (2008).

[64] P. Romatschke and U. Romatschke, Viscosity Information from Relativistic Nuclear Collisions: How Perfect is the Fluid Observed at RHIC? Phys. Rev. Lett. 99, 172301 (2007).

[65] H. Li, Xin-li Sheng, and Q. Wang, Electromagnetic fields with electric and chiral magnetic conductivities in heavy ion collisions, Phys. Rev. C 94, 044903 (2016).

[66] Y. Zhong, Yang C. B., X. Cai, and S.-Q. Feng, Spatial distributions of magnetic field in the RHIC and LHC energy regions, Chin. Phys. C 39, 104105 (2015).

[67] S. Pu, V. Roy, L. Rezzolla, and D. H. Rischke, Bjorken flow in one-dimensional relativistic magnetohydrodynamics with magnetization, Phys. Rev. D 93, 074022 (2016).

[68] D. She, Z. F. Jiang, D. Hou, and C. B. Yang, $1+1$ dimensional relativistic magnetohydrodynamics with longitudinal acceleration, Phys. Rev. D 100, 116014 (2019).

[69] S. Cao, T. Luo, G.-Y. Qin, and X.-N. Wang, Linearized Boltzmann transport model for jet propagation in the quark-gluon plasma: Heavy quark evolution, Phys. Rev. C 94, 014909 (2016).

[70] C. A. G. Prado, W.-J. Xing, S. Cao, G.-Y. Qin, and X.-N. Wang, Longitudinal dependence of open heavy flavor $R_{\mathrm{AA}}$ in relativistic heavy-ion collisions, Phys. Rev. C 101, 064907 (2020).

[71] W.-J. Xing, S. Cao, G.-Y. Qin, and H. Xing, Flavor hierarchy of jet quenching in relativistic heavy-ion collisions, Phys. Lett. B 805, 135424 (2020). 
[72] S.-Q. Li, W.-J. Xing, F.-L. Liu, S. Cao, and G.-Y. Qin, Heavy flavor quenching and flow: The roles of initial condition, preequilibrium evolution, and in-medium interaction, Chin. Phys. C 44, 114101 (2020).

[73] S. A. Bass et al., Microscopic models for ultrarelativistic heavy ion collisions, Prog. Part. Nucl. Phys. 41, 255 (1998).

[74] W. Zhao, W. Ke, W. Chen, T. Luo, and X.-N. Wang, From hydro to jet quenching, coalescence and hadron cas- cade: A coupled approach to solving the $R_{A A} \otimes v_{2}$ puzzle, arXiv:2103.14657.

[75] H. Petersen, D. Oliinychenko, M. Mayer, J. Staudenmaier, and S. Ryu, SMASH-A new hadronic transport approach, Nucl. Phys. A 982, 399 (2019).

[76] X.-Y. Wu, G.-Y. Qin, L.-G. Pang, and X.-N. Wang, $(3+1)-D$ viscous hydrodynamics CLVisc at finite net baryon density: identified particle sspectra, anisotropic flows and flow fluctuations across BES energies, arXiv:2107.04949. 